\title{
A neural interlingua for multilingual machine translation
}

\author{
Yichao Lu*, Phillip Keung*, Faisal Ladhak, Vikas Bhardwaj, Shaonan Zhang, Jason Sun \\ \{yichaolu, keung, faisall, vikab, shaonanz, jasun\}@amazon.com \\ Amazon Inc.
}

\begin{abstract}
We incorporate an explicit neural interlingua into a multilingual encoder-decoder neural machine translation (NMT) architecture. We demonstrate that our model learns a languageindependent representation by performing direct zero-shot translation (without using pivot translation), and by using the source sentence embeddings to create an English Yelp review classifier that, through the mediation of the neural interlingua, can also classify French and German reviews. Furthermore, we show that, despite using a smaller number of parameters than a pairwise collection of bilingual NMT models, our approach produces comparable BLEU scores for each language pair in WMT15.
\end{abstract}

\section{Introduction}

\subsection{Multilingual Machine Translation}

Neural machine translation (NMT) relies on word and sentence embeddings to encode the semantic information needed for translation. The standard attentional encoder-decoder models (Bahdanau et al., 2015) for bilingual NMT decompose naturally into separate encoder and decoder subnetworks for the source and target languages. This factorization has inspired various forms of multilingual NMT models that extended the original bilingual framework to handle more language pairs simultaneously. We refer to NMT models that accept sentences from one source language and produce outputs in one target language as 'bilingual'. We contrast this with 'multilingual' NMT models, which support more than one source and/or target languages within the same model.

The naive approach to multilingual machine translation would train a model for each language pair, which scales quadratically with the number

\footnotetext{
* Equal contribution
}

of languages in the corpus. Instead, by combining language-specific encoders and decoders in different ways, Dong et al. (2015), Zoph and Knight (2016), Luong et al. (2016), and Firat et al. (2016a) have explored the one source-tomany target, many source-to-one target, and many source-to-many target multilingual MT settings. The multi-way shared attention model (Firat et al., 2016a) is closest to our work, in that they consider the large-scale, many-to-many scenario with multiple encoders and decoders.

It is also possible to adapt existing bilingual NMT models to the many-to-many case without changing the architecture at all. The universal encoder-decoder approach (Ha et al., 2016; Johnson et al., 2017) constructs a shared vocabulary for all languages in the dataset, and use just one encoder and decoder for multilingual translation. In addition, Johnson et al. (2017) introduce direct zero-shot translation, which refers to the task of translating between language pairs without parallel text or pivoting through an intermediate language like English. Direct zero-shot translation may yield lower BLEU scores than pivot-based approaches, but avoids doubling the latency and computational overhead (due to translating the source sentence twice,) which is a concern for large-scale, productionized MT systems.

Nonetheless, both the multi-way shared attention model and the universal encoder-decoder model suffer from certain disadvantages. For the former, direct zero-shot translation was shown to be impossible in Firat et al. (2016b), and there is no indication that the model learns any kind of shared representation across languages. For the latter, the output vocabulary size is typically fixed to the vocabulary size for a single target language (i.e. roughly 20,000 to 30,000 types), regardless of the number of languages in the corpus. Increasing the vocabulary size is costly, since the training 
and inference time scales linearly with the size of the decoder's output layer.

\subsection{Our Contributions}

In this work, we construct an explicit neural interlingua for multilingual NMT, which addresses some of the limitations in existing approaches. Our contributions are threefold:

Firstly, we describe an attentional neural interlingua that receives language-specific encoder embeddings and produces output embeddings which are agnostic to the source and target languages.

Secondly, we perform zero-shot translation (without pivot translation) for the $\mathrm{Fr} \leftrightarrow \mathrm{Ru}, \mathrm{Zh} \leftrightarrow \mathrm{Es}$ and Es $\leftrightarrow$ Fr pairs of the updated UN Parallel Corpus (Ziemski et al., 2016). At the time of writing, our approach is the only alternative to the universal encoder-decoder model for direct neural zero-shot translation. We observe a significant improvement in zero-shot translation performance compared to that model.

Finally, we demonstrate that our model generates useful representations for crosslingual transfer learning. We use the source sentence embeddings from our translation model to create an English Yelp review classifier that can, through the mediation of the interlingua, classify French and German Yelp reviews. We also show that the sentence embeddings of parallel translations are close to each other in a low-dimensional space.

\section{Model Architecture}

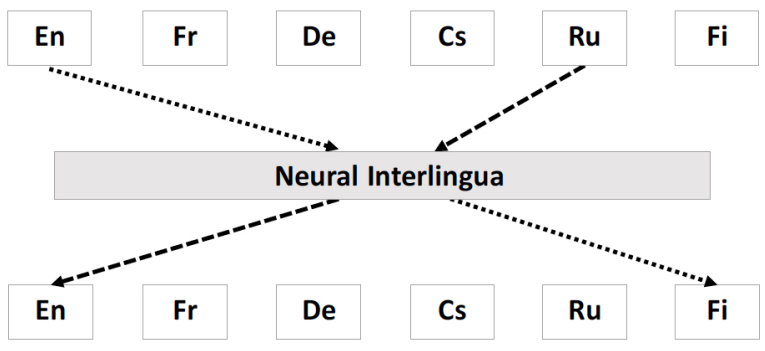

Figure 1: Our encoder-decoder model with the neural interlingua, trained on WMT15. The neural interlingua is an attentional encoder that converts languagespecific embeddings to language-independent ones. Here, we illustrate the flow of data from English $\rightarrow$ Interlingua $\rightarrow$ Finnish, and Russian $\rightarrow$ Interlingua $\rightarrow$ English.

Figure 1 illustrates our basic model architecture. Each language has its own recurrent encoder and decoder. We attempt to construct a neural interlin-

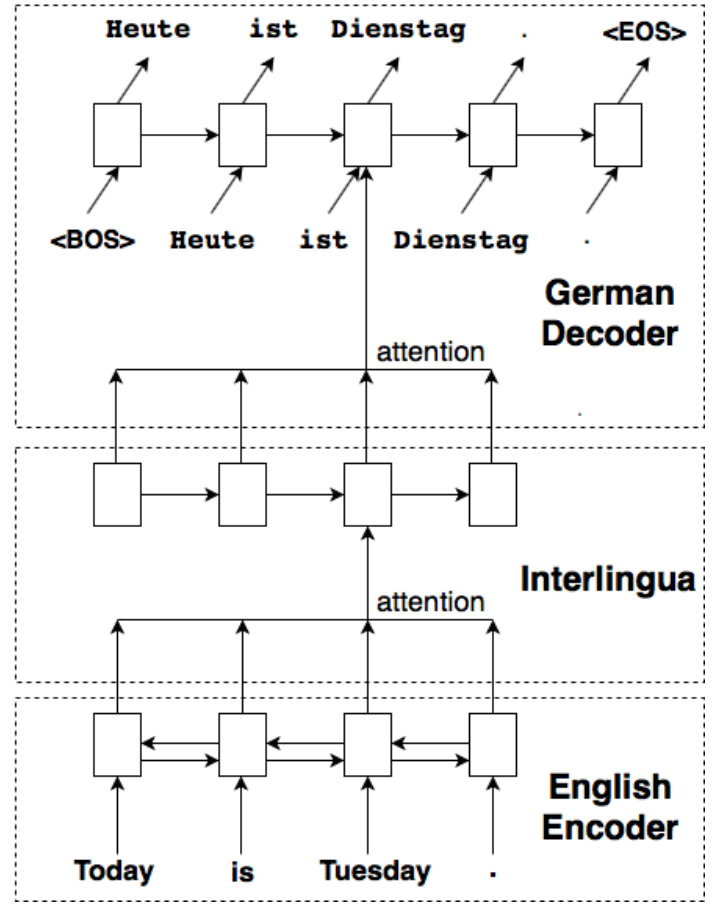

Figure 2: An in-depth look at the network structure when training/predicting with an En-De batch. The English sentence is fed through the English bidirectional LSTM encoder. The encoder states are passed into the neural interlingua, which is an attentional LSTM encoder. Finally, the hidden states of the interlingua are consumed by the German attentional LSTM decoder to generate the German translation.

gua by passing the language-specific encoder embeddings through a shared recurrent layer, whose output embeddings are then passed to languagespecific decoders.

The figure describes the flow of data in the model; each minibatch only contains one source language and one target language, and only the parameters in the source encoder, interlingua, and target decoder are used for the forward and backward passes. During training, the source and target languages in each minibatch rotate according to a schedule (see Algorithm 1). In Figure 2, we illustrate how an English sentence is converted into a German one.

As with most sequence-to-sequence models, we can view the generation of the next token in the target sentence as the application of a series of neural network operations on the source sentence and the partial output thus far. We model the probability of each target sentence as follows, 


$$
\begin{gathered}
p\left(y_{i} \mid y_{<i}, x\right)=\operatorname{Dec}_{t}\left(\operatorname{Inter}\left(\operatorname{Enc}_{s}\left(\operatorname{Emb}_{s}(x)\right)\right),\right. \\
\left.y_{i-1}, h_{i-1}^{t}\right)
\end{gathered}
$$

where $y$ is the target sentence, $x$ is the source sentence, Dec $_{t}$ is the decoder for the target language $t$, Inter is the neural interlingua, Enc ${ }_{s}$ is the encoder for the source language $s, \mathrm{Emb}_{s}$ is the word embedding matrix for $s, h_{i-1}^{t}$ is the state of the decoder at step $i-1, s \in\{1, \ldots, S\}$ is the index of the source language, and $t \in\{1, \ldots, T\}$ is the index of the target language.

The source sentence $x$ is transformed from a sequence of one-hot representations to a sequence of word embeddings $B^{s}$ through $\mathrm{Emb}_{s}$,

$$
B^{s}=\operatorname{Emb}_{s}\left(x^{s}\right)
$$

$B^{s}$ is a $b^{s} \times L_{x}$ matrix, where $L_{x}$ is the length of the source sentence, and $b^{s}$ is the size of the word embedding for the source language $s$.

The sequence of word embeddings is converted into a sentence representation $E^{s}$ by $\mathrm{Enc}_{s}$,

$$
\begin{aligned}
E_{., i}^{s} & =\operatorname{Enc}_{s}\left(B^{s}\right)_{., i} \\
& =\operatorname{BiLSTM}\left(B_{., i}^{s}, h_{i-1}^{s}\right)
\end{aligned}
$$

$E^{s}$ is a $e^{s} \times L_{x}$ matrix, where $e^{s}$ is the size of encoder's output. The notation $X_{., i}$ refers to the $i^{\text {th }}$ column of the matrix $X$. BiLSTM is a bidirectional LSTM network, with forward and backward states $h_{i-1}^{s}=\left[\vec{h}_{i-1}^{s}, \overleftarrow{h}_{i+1}^{s}\right]$ for step $i-1$.

The neural interlingua Inter is an attentional encoder that maps the language-specific representation $E^{s}$ to an interlingual representation $I$,

$$
\begin{aligned}
I_{., i} & =\operatorname{Inter}\left(E^{s}\right)_{., i} \\
& =W^{I}\left[\operatorname{LSTM}\left(c_{i}^{I}, h_{i-1}^{I}\right), c_{i}^{I}\right]+b^{I} \\
& =W^{I}\left[h_{i}^{I}, c_{i}^{I}\right]+b^{I}
\end{aligned}
$$

where $h_{i-1}^{I}$ is the interlingua LSTM state for step $i-1, c_{i}^{I}=\sum_{j=1}^{L_{x}} \alpha_{i j}^{I} E_{., j}^{s}$ is the attentional context vector, $\alpha_{i j}^{I}=\frac{\exp \left(e_{i j}^{I}\right)}{\sum_{j} \exp \left(e_{i j}^{I}\right)}$ and $e_{i j}^{I}=$ $\operatorname{MLP}_{I}\left(h_{i}^{I}, E_{,, j}^{s}\right)$ are the normalized and unnormalized attention weights introduced in Bahdanau et al. (2015), and $z=[x, y]$ denotes the concatenation of the vectors $x$ and $y$ into a new vector $z$. We perform an affine transformation with $W^{I}, b^{I}$ to project the interlingua output to the desired dimensions.

$I$ is a $e^{i} \times L_{i}$ matrix, where $e^{i}$ is the size of the interlingua's output. The output of the neural interlingua is always fixed in length to $L_{i}$ (where $L_{i}=50$ in our experiments), regardless of the length of the source sentence. We chose $L_{i}=50$ because, during model training, we restrict the maximum source sentence length to 50 . To avoid learning language-specific embeddings, we do not use indicator tokens for the source or target languages.

Finally, the decoder takes the interlingual representation $I$ and the partial target sentence $y_{<i}$ and computes the probability distribution for the next output token,

$$
\begin{aligned}
& p\left(y_{i} \mid y_{<i}, x\right) \\
& =\operatorname{Dec}_{t}\left(I, y_{i-1}, h_{i-1}^{t}\right)_{., i} \\
& =\operatorname{softmax}\left(W^{t}\left[\operatorname{LSTM}\left(\left[y_{i-1}, c_{i}^{t}\right], h_{i-1}^{t}\right), c_{i}^{t}\right]+b^{t}\right) \\
& =\operatorname{softmax}\left(W^{t}\left[h_{i}^{t}, c_{i}^{t}\right]+b^{t}\right)
\end{aligned}
$$

where $c_{i}^{t}=\sum_{j=1}^{L_{i}} \alpha_{i j}^{t} I_{., j}$ is the context vector at step $i$, and $\alpha_{i j}^{t}$ are the normalized attention weights. The decoders receive the source sentence only through the interlingual embedding.

Like Firat et al. (2016a), the number of encoders and decoders for our model architecture scales linearly (rather than quadratically) with the number of languages. In addition, since the neural interlingua provides a common source sentence representation to all decoders, the number of attention mechanisms also scales linearly with the number of languages.

We note that the concept of a neural interlingua is independent of the architecture that is chosen. While we use a LSTM encoder-decoder model with single-headed attention for experimental simplicity, one could also introduce a neural interlingua to a transformer network (Vaswani et al., 2017) or a CNN encoder-decoder network (Gehring et al., 2017) instead.

\section{Experiments}

We conducted 4 experiments with our model.

We compared the performance of bilingual NMT baselines against our proposed multilingual model, and observe comparable performance across all the language pairs in WMT15. 


\begin{tabular}{|c|c|c|}
\hline Parameter & $\begin{array}{c}\text { Multi } \\
\text {-lingual }\end{array}$ & Bilingual \\
\hline vocabulary size & 30,000 & 30,000 \\
source embedding size & 256 & 256 \\
target embedding size & 256 & 256 \\
output dimension & 512 & 512 \\
encoder hidden size & 512 & 512 \\
decoder hidden size & 512 & 512 \\
interlingua hidden size & 512 & - \\
interlingua length & 50 & - \\
encoder depth & 2 & 4 \\
interlingua depth & 1 & 0 \\
decoder depth & 1 & 1 \\
attention type & additive & additive \\
optimizer & Adam & Adam \\
learning rate & 0.0002 & 0.0002 \\
batch size & 400 & 400 \\
\hline
\end{tabular}

Table 1: Hyperparameters for the multilingual and bilingual encoder-decoder models.

We found that the language-independent sentence embeddings can be used for zero-shot multilingual classification. We train an English Yelp review classifier with the interlingual embeddings as input features, and use that model to classify French and German reviews.

We performed direct zero-shot translation for 3 language pairs in the new UN Parallel Corpus. For this task, our model showed an improvement over the model architecture described in Johnson et al. (2017). Our positive experimental finding confirms that our model provides a new approach for direct neural zero-shot translation.

Finally, we visualized the languageindependent sentence embeddings by projecting them down to 2 dimensions. We observe that parallel translations of French, German and English sentences remain close to each other in this low-dimensional space.

\subsection{Model Training}

The hyperparameters for the bilingual baseline models and our multilingual network are summarized in Table 1. Our multilingual model uses 1 bidirectional LSTM layer in the encoder for each input language, 1 attentional LSTM layer for the interlingua and 1 attentional LSTM layer in the decoder for each output language. The baseline bilingual models use 2 bidirectional LSTM layers in the encoder and 1 attentional LSTM layer in the decoder. We chose the Adam optimizer (Kingma and $\mathrm{Ba}, 2015$ ), and we used importance sampling, as described in Jean et al. (2015), to accelerate model training.

\subsection{Language Rotation During Training}

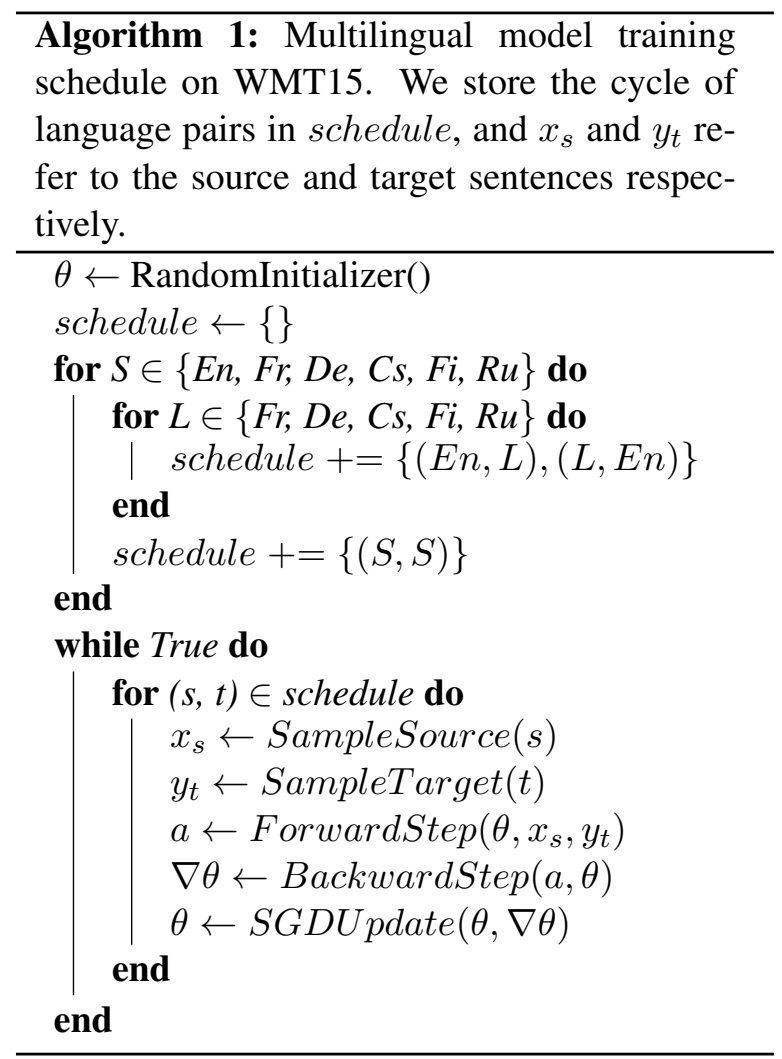

The language pair schedule used during training is crucial for learning an effective sentence representation. We provide the details in Algorithm 1. In our initial experiments, we cycled through 10 language pairs (i.e. $(x \rightarrow$ En, En $\rightarrow$ $x), x \in\{\mathrm{Fr}, \mathrm{De}, \mathrm{Ru}, \mathrm{Cs}, \mathrm{Fi}\}$ ), where each minibatch consisted of sentences from one language pair. However, we found that the naive schedule failed to produce a useful representation for zeroshot translation or crosslingual text classification. Since WMT15 is not a multi-parallel corpus, the model essentially learns to handle two separate tasks, namely translation from English and translation to English. For instance, since the output of the De encoder and the En encoder would never be used by the same decoder, there is no reason for De and En source sentences to share the same embedding, even if they are translations of each other.

To encourage the model to share the encoder representations across English and non-English 


\begin{tabular}{|c|c|c|c|}
\hline Source & Target & Bilingual & Multilingual \\
\hline & $\mathrm{Fr}$ & $\mathbf{3 4 . 8 5}$ & 33.80 \\
& $\mathrm{De}$ & $\mathbf{2 3 . 6 7}$ & 23.37 \\
En & $\mathrm{Cs}$ & $\mathbf{1 7 . 6 0}$ & 16.62 \\
& $\mathrm{Ru}$ & 21.26 & $\mathbf{2 1 . 9 2}$ \\
& $\mathrm{Fi}$ & 11.55 & $\mathbf{1 3 . 3 4}$ \\
\hline $\mathrm{Fr}$ & & $\mathbf{3 0 . 7 2}$ & 30.24 \\
$\mathrm{De}$ & & 27.08 & $\mathbf{2 7 . 2 9}$ \\
$\mathrm{Cs}$ & $\mathrm{En}$ & 23.00 & $\mathbf{2 3 . 8 7}$ \\
$\mathrm{Ru}$ & & 24.14 & $\mathbf{2 6 . 1 5}$ \\
$\mathrm{Fi}$ & & 14.77 & $\mathbf{1 6 . 5 8}$ \\
\hline
\end{tabular}

Table 2: Comparison of BLEU scores across language pairs in newstest 2015 and newsdiscuss 2015 . We show the results for the bilingual baseline NMT models and our multilingual NMT model.

source sentences, we added an extra identity language pair (i.e. $\mathrm{De} \rightarrow \mathrm{De}, \mathrm{En} \rightarrow \mathrm{En}$, etc.) to the rotation. The identity pair forces the source embeddings to be compatible with an additional decoder. We found that when we did not include the identity mapping task during training, the zero-shot BLEU score was $<1.0$ for the Fr-Ru language pair.

\subsection{Multilingual NMT versus Bilingual NMT}

We used the training corpora from the WMT15 translation task to train our encoder-decoder models. The dataset provides English $\leftrightarrow$ (German, French, Czech, Russian, Finnish) parallel sentences. We followed the standard WMT preprocessing recipes ${ }^{1}$, which are based on the Moses library (Koehn et al., 2007). For each language, we created a vocabulary of 30,000 word pieces using byte pair encoding (Sennrich et al., 2016). Sentences longer than 50 word pieces were removed from the training corpus. We used newstest2014 and newsdev2015 as our development set, and newstest 2015 and newsdiscuss 2015 as our test set.

We compared the performance of the multilingual model against bilingual baseline models. The BLEU scores are provided in Table 2. Results are reported on newstest2015 and newsdiscuss 2015. We see that, while the performance is broadly similar (i.e. generally $<1.0$ BLEU) between the our model and the baselines, there is a decrease in BLEU for higher-resource languages (e.g. Fr) and an increase in BLEU for lower-resource languages

\footnotetext{
1e.g. http://data.statmt.org/wmt17/ translation-task/preprocessed/de-en/ prepare.sh
}

(e.g. $\mathrm{Fi}, \mathrm{Ru}$ ). We suspect that this is a consequence of the language pair schedule, which cycles through all language pairs as though they were equally frequent in the corpus. A similar effect was also observed in Johnson et al. (2017).

Currey et al. (2017) have shown that (specifically in low-resource settings) using copied monolingual data can improve model performance. We followed the technique in Currey et al. (2017) to strengthen the baseline models, but did not observe an improvement in the final BLEU score. This may be due to the fact that even the smallest language pair in WMT15 has 2 million sentence pairs, which is more than 3 times larger than either the Tr-En or Ro-En pairs discussed in Currey et al. (2017).

As with Firat et al. (2016a), we generally see an improvement when translating to English. We believe that this is because the English language model is stronger in the multilingual case, since the English decoder sees more English text.

\subsection{Zero-shot Multilingual Classification}

We constructed a multilingual Yelp review dataset from a subset of the Yelp Challenge (Round 10) corpus. We restrict ourselves to English, French, and German reviews. The training corpus consists of 5,000 English Yelp reviews, and the test sets contain 4,000 reviews for each language. The French and German reviews were extracted by applying language detection on reviews from Quebec, Canada and Baden-Württemberg, Germany. The review scores were binarized, where 4 and 5 star reviews were labeled as positive, and 1 and 2 star reviews were labeled as negative. We reuse the encoders trained in Section 3.3 in this section's experiments.

At training time, an English Yelp review is treated as one sentence; we do not apply sentence segmentation to the review. It is passed through the English encoder, and the neural interlingua converts the English sentence representation to a fixed-length representation. To create a feature vector for the text classifier, we apply mean-pooling to the sentence representation. Under our experimental settings, every sentence is converted to a $512 \times 50$ interlingual embedding, which is mean-pooled into a 512-dimensional vector. We then fit a logistic regression model using this feature vector and the sentence polarity as the binary label. The classifier is only trained on En- 


\begin{tabular}{|c|c|c|}
\hline Color & Lang. & Text \\
\hline Green & En & $\begin{array}{l}\text { spreads between sovereign bonds in Germany and those in other countries were relatively unaffected } \\
\text { by political and market uncertainties concerning Greece in late } 2014 \text { and early } 2015 \text {. } \\
\text { par contre, la différence entre les obligations souveraines allemandes et celles d'autres pays a été } \\
\text { relativement peu touchée par les incertitudes politiques et les doutes des marchés concernant la Grèce } \\
\text { fin } 2014 \text { et début } 2015 \text {. } \\
\text { политическая и рыночная нестабильность , связанная с ситуацией в Греции в конце } \\
2014 \text { - го и начале } 2015 \text { года , практически не отразилась на спредах доходности между } \\
\text { государственными облигациями Германии и других стран . }\end{array}$ \\
\hline Red & En & $\begin{array}{l}13 \text {. we underscore the need to accelerate efforts at all levels to achieve the objectives of the international } \\
\text { arrangement on forests beyond } 2015 \text { and the need to establish a stronger, more effective and solid } \\
\text { arrangement for the period } 2015 \text { to } 2030 \text {; } \\
13 \text {. nous soulignons qu'il faudra redoubler d'efforts à tous les niveaux pour atteindre les objectifs de } \\
\text { l'arrangement international après } 2015 \text { et qu'il faudra mettre en place un arrangement plus solide et } \\
\text { plus efficace pour la période } 2015 \text { - } 2030 \text {; } \\
13 \text {. мы подчеркиваем, что необходимо активизировать усилия на всех уровнях в } \\
\text { интересах достижения целей международного механизма по лесам на период после } 2015 \\
\text { года и создать действенный , более эффективный и надежный механизм на период } 2015 \\
\text { - 2030 годов ; }\end{array}$ \\
\hline Orange & $\begin{array}{l}\mathrm{En} \\
\mathrm{Fr} \\
\mathrm{Ru}\end{array}$ & $\begin{array}{l}\text { the various training activities are listed in table } 2 \text { below . } \\
\text { on énumère dans le tableau } 2 \text { ci - dessous les diverses activités de formation. } \\
\text { в представленной далее таблице } 2 \text { приведен перечень различных мероприятий по } \\
\text { профессиональной подготовке. }\end{array}$ \\
\hline Blue & $\begin{array}{l}\text { En } \\
\mathrm{Fr} \\
\mathrm{Ru}\end{array}$ & $\begin{array}{l}\text { the Conference affirms that, pending the realization of this objective, it is in the interest of the very } \\
\text { survival of humanity that nuclear weapons never be used again. } \\
\text { elle affirme que, en attendant la réalisation de cet objectif, il est dans l'intérêt de la survie même de } \\
\text { l'humanité que les armes nucléaires ne soient plus jamais utilisées . } \\
\text { конференция заявляет, что, пока эта цель не достигнута, необходимо в интересах } \\
\text { самого выживания человечества добиться того , чтобы ядерное оружие никогда не } \\
\text { было вновь применено. }\end{array}$ \\
\hline
\end{tabular}

Table 3: Text of the parallel sentences in Figure 3.

\begin{tabular}{|c|c|c|c|}
\hline \multirow{2}{*}{} & \multicolumn{3}{|c|}{ Input Language } \\
\cline { 2 - 4 } & En & De & Fr \\
\hline Trigram & $91.6 \% \pm 0.9 \%$ & $89.6 \% \pm 0.9 \%$ & $91.5 \% \pm 0.9 \%$ \\
\hline Embeddings & $91.5 \% \pm 0.9 \%$ & $89.2 \% \pm 0.9 \%$ & $91.1 \% \pm 0.9 \%$ \\
\hline$\%$ Positive & $82.9 \%$ & $86.7 \%$ & $88.5 \%$ \\
\hline
\end{tabular}

Table 4: Accuracy for crosslingual Yelp binary review classification. The trigram baseline model was trained on English reviews, and tested on English reviews and English translations of French and German reviews. The embedding-based classifier uses interlingual embeddings from our model in Section 3.3. '\% Positive' refers to the proportion of the test set that has a positive label.

glish reviews.

At prediction time, we pass the text of a German review through the German encoder and the interlingua, which is again mean-pooled to form a 512dimensional vector. Since the interlingual representation should be language-independent, we can attempt to classify German reviews by providing the vector representation of the German review to the English classifier. We use the same process for French reviews.

In Table 4, we compare the accuracy of the classifier trained on English review embeddings to that of a baseline model. We established the baseline by training a trigram classifier on the English reviews, and used English translations of the French and German reviews for classification. We ob- tained the translations through the Google Translate API. The classification accuracy using the interlingual embeddings or the translated French and German reviews are similar, which shows that the embeddings have retained semantic information in a language-independent way.

\subsection{Direct Zero-shot Translation}

The updated UN Parallel Corpus (Ziemski et al., 2016), unlike the WMT corpus, is a fully multiparallel corpus that contains English, Spanish, French, Arabic, Chinese and Russian text. We used this corpus as a testbed for our zero-shot translation experiments.

We trained our multilingual model on the UN corpus, following the same settings that we used 


\begin{tabular}{|l|r|r|r|r|r|r|}
\hline & Fr-Ru & Ru-Fr & Es-Zh & Zh-Es & Es-Fr & Fr-Es \\
\hline This Work & 18.24 & 21.61 & 17.66 & 18.66 & 30.08 & 31.94 \\
\hline Univ. Enc-Dec & 8.77 & 9.76 & 8.62 & 6.13 & 15.04 & 14.37 \\
\hline Pivot & 20.87 & 27.34 & 26.03 & 26.01 & 31.84 & 32.93 \\
\hline Direct NMT & 28.29 & 33.26 & 32.36 & 32.69 & 41.38 & 44.49 \\
\hline
\end{tabular}

Table 5: Zero-shot BLEU scores on the UN Parallel Corpus on selected language pairs. The universal encoderdecoder, pivot and direct NMT results were retrieved from Miura et al. (2017). Our proposed model outperforms the universal encoder-decoder model (Johnson et al., 2017) on the zero-shot translation task.

for the WMT corpus (see Table 1 and Algorithm 1). The text was processed following the steps provided in Miura et al. (2017). We restrict the training corpus to sentence pairs that have English as either the source or target language.

We used the Fr-Ru, Es-Zh and Es-Fr portions of the test set from the UN corpus for the zero-shot translation evaluation. The training dataset that we constructed does not contain direct Fr-Ru, Es-Zh or Es-Fr sentence pairs. The test set contains 4,000 sentence pairs for each language pair.

We examine the BLEU scores for zero-shot translation on the UN corpus in Table 5. The universal encoder-decoder, pivot and direct NMT results were retrieved from (Miura et al., 2017). By 'direct NMT', we refer to a model trained directly on the parallel text.

Our multilingual model performs significantly better on the direct zero-shot task than the universal encoder-decoder approach of Johnson et al. (2017). Generally, our model does not perform as well as the pivot approach, though in the case of Es-Fr and Fr-Es, the difference is surprisingly small ( $<2.0$ BLEU).

Improving direct zero-shot methods to reach parity with pivot translation has practical consequences for large-scale NMT systems, like reduced latency and computational overhead. (Recall that pivot translation must translate every source sentence twice; first into the intermediate language, and then into the target language.) Our results show progress towards the goal of transitioning away from pivot-based methods to neural zero-shot translation.

\subsection{Interlingua Visualization}

In Figure 3, we plot the embeddings for 4 groups of parallel sentences. Sentences from the same group share the same color. Each group contains one French, one English and one Russian sentence which are parallel translations of each other. We

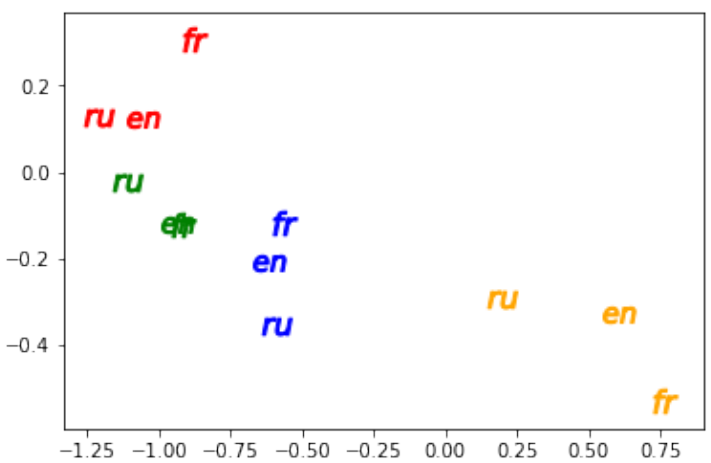

Figure 3: Interlingual embeddings for four groups of parallel English, French, and Russian sentences from the UN Parallel Corpus. The 512-dimensional meanpooled interlingual sentence embeddings were projected down to $\mathbf{R}^{2}$ using PCA. Refer to Table 3 for the colors and text of the sentences.

provide the text of the embedded sentences in Table 3 .

The embeddings were generated by meanpooling each sentence embedding to a 512dimensional vector and projecting it to $\mathbf{R}^{2}$ using PCA. From the figure, we observe a clear separation between different groups of sentences, while sentences within the same group remain close to each other in space. This is the expected outcome if our model has captured language-independent semantic information in its sentence representations.

\section{Related Work}

\subsection{Networks with Language-specific Encoders and Decoders}

The many-to-one approach explored in Zoph and Knight (2016) primarily considers the trilingual case, where a multi-parallel corpus is available, and uses 2 encoders simultaneously to provide the source context for the decoder. We note that using 2 encoders simultaneously requires having 2 source sentences for every desired target sentence 
at prediction time, which is not the setting that we investigate here.

By combining a single encoder with multiple attentional decoders, the one-to-many approach presented in Dong et al. (2015) showed an improvement in translation performance, due to the increase in the number of sentences seen by the encoder and through multi-task learning.

The many-to-many approach in the shared attention model (Firat et al., 2016a) assigns a different encoder and decoder to each language, but shares the decoders' attention mechanisms. By specifying a 'universal' attention mechanism for all language pairs, Firat et al. (2016a) avoid creating as many attention mechanisms as there are language pairs (i.e. avoids quadratic scaling).

However, the attention mechanism acts as the alignment model between the source and target sentences, and a shared attention mechanism may be too restrictive, especially for languages that have very different word orders. Our interlingual approach relaxes the requirement of a single, shared attention mechanism. In our framework, there are as many attention mechanisms as there are decoders.

\subsection{Universal Encoder-Decoder Networks}

Johnson et al. (2017) have foregone the use of multiple encoders and decoders, and instead use one universal encoder and one universal decoder. They constructed a joint vocabulary for all languages in the corpus, consisting of word pieces derived from a byte-pair encoding (Sennrich et al., 2016) on the union of the vocabulary of all the languages, and include special tokens to indicate what the output language should be. Ha et al. (2016) follow a similar approach, but the shared vocabulary is constructed by prepending a language identifier to each token.

The universal encoder-decoder approach does have some shortcomings. Johnson et al. (2017) rely on the existence of a shared vocabulary, which may not be as sensible in some combinations (e.g. Chinese and English) as in others (e.g. Spanish and Portuguese). If the languages' vocabularies do not share many word pieces, then either the decoder's output layer will be very large, which slows down training and inference, or the output layer will be artificially constrained to a manageable size, which impacts translation performance.

Our approach, on the other hand, allows each target language to retain its own decoder. The total vocabulary size can then expand with the number of languages without affecting training or inference speed.

\subsection{Zero-shot Translation}

One of the challenges in multilingual MT is data sparsity, which refers to the lack of parallel text for every possible language pair in a corpus. Zeroshot translation is the task of translating between language pairs without parallel text.

An early approach to allow zero-shot translation made use of a 'pivot' language in the translation process (Boitet, 1988). For instance, in sentencebased pivoting, the source sentence is translated into a pivot language, and from the pivot language translated to the target language. Various extensions of the pivot technique have been proposed over the years, see Utiyama and Isahara (2007), Chen et al. (2017), Miura et al. (2017), Cohn and Lapata (2007).

Universal encoder-decoder systems like Johnson et al. (2017) have demonstrated the ability to perform direct zero-shot translation without using a pivot language at all, albeit with a significant BLEU reduction for some language pairs.

\section{Conclusion}

We incorporate a neural interlingua component into the standard encoder-decoder framework for multilingual neural machine translation, and demonstrate that the resulting model learns language-independent sentence representations, enabling zero-shot translation and crosslingual text classification.

We perform direct zero-shot translation for 3 language pairs without pivoting through an intermediate language like English. We observe an improvement in zero-shot translation performance compared to the universal encoder-decoder results reported in Miura et al. (2017). Furthermore, we use the learned encoder to train an English Yelp review classifier that can, with the help of the interlingual embeddings, also classify German and French reviews. Finally, our experiments showed that the results from our model are comparable to the results from bilingual baselines.

In future work, we intend to address the significant performance gap between direct neural zeroshot translation and pivot translation. By manipulating the sentence embeddings in an appropriate 
way, we aim to extract significant improvements over the results presented in this paper.

\section{References}

Dzmitry Bahdanau, Kyunghyun Cho, and Yoshua Bengio. 2015. Neural machine translation by jointly learning to align and translate. In ICLR.

Christian Boitet. 1988. Pros and cons of the pivot and transfer approaches in multilingual machine translation. In Readings in machine translation, pages 273-279.

Yun Chen, Yang Liu, Yong Cheng, and Victor OK Li. 2017. A teacher-student framework for zeroresource neural machine translation. $A C L$.

Trevor Cohn and Mirella Lapata. 2007. Machine translation by triangulation: Making effective use of multi-parallel corpora. In $A C L$.

Anna Currey, Antonio Valerio Miceli Barone, and Kenneth Heafield. 2017. Copied monolingual data improves low-resource neural machine translation. In WMT.

Daxiang Dong, Hua Wu, Wei He, Dianhai Yu, and Haifeng Wang. 2015. Multi-task learning for multiple language translation. In $A C L$.

Orhan Firat, Kyunghyun Cho, and Yoshua Bengio. 2016a. Multi-way, multilingual neural machine translation with a shared attention mechanism. In NAACL-HLT.

Orhan Firat, Baskaran Sankaran, Yaser Al-Onaizan, Fatos T Yarman Vural, and Kyunghyun Cho. 2016b. Zero-resource translation with multi-lingual neural machine translation. In EMNLP.

Jonas Gehring, Michael Auli, David Grangier, Denis Yarats, and Yann N Dauphin. 2017. Convolutional sequence to sequence learning. In ICML.

Thanh-Le Ha, Jan Niehues, and Alexander Waibel. 2016. Toward multilingual neural machine translation with universal encoder and decoder. In IWSLT.

Sébastien Jean, Kyunghyun Cho, Roland Memisevic, and Yoshua Bengio. 2015. On using very large target vocabulary for neural machine translation. In $A C L$.

Melvin Johnson, Mike Schuster, Quoc V Le, Maxim Krikun, Yonghui Wu, Zhifeng Chen, Nikhil Thorat, Fernanda Viégas, Martin Wattenberg, Greg Corrado, et al. 2017. Google's multilingual neural machine translation system: enabling zero-shot translation. In $T A C L$

Diederik Kingma and Jimmy Ba. 2015. Adam: A method for stochastic optimization. In ICLR.
Philipp Koehn, Hieu Hoang, Alexandra Birch, Chris Callison-Burch, Marcello Federico, Nicola Bertoldi, Brooke Cowan, Wade Shen, Christine Moran, Richard Zens, et al. 2007. Moses: Open source toolkit for statistical machine translation. In $A C L$.

Minh-Thang Luong, Quoc V Le, Ilya Sutskever, Oriol Vinyals, and Lukasz Kaiser. 2016. Multi-task sequence to sequence learning. In ICLR.

Akiva Miura, Graham Neubig, Katsuhito Sudoh, and Satoshi Nakamura. 2017. Tree as a pivot: Syntactic matching methods in pivot translation. In WMT.

Rico Sennrich, Barry Haddow, and Alexandra Birch. 2016. Neural machine translation of rare words with subword units. In $A C L$.

Masao Utiyama and Hitoshi Isahara. 2007. A comparison of pivot methods for phrase-based statistical machine translation. In NAACL-HLT.

Ashish Vaswani, Noam Shazeer, Niki Parmar, Jakob Uszkoreit, Llion Jones, Aidan N Gomez, Lukasz Kaiser, and Illia Polosukhin. 2017. Attention is all you need. In NIPS.

Michal Ziemski, Marcin Junczys-Dowmunt, and Bruno Pouliquen. 2016. The united nations parallel corpus v1. 0. In $L R E C$.

Barret Zoph and Kevin Knight. 2016. Multi-source neural translation. In NAACL-HLT. 